\title{
Remoção cirúrgica de nódulos de Spirocerca lupi
}

\author{
Surgical removal of the granuloma of Spirocerca lupi \\ Ana Maria Quessada ${ }^{1}$, Francisco Lima Silva ${ }^{1}$, \\ André Braga de Souza² \& Antonio Augusto Rodrigues de Sousa ${ }^{3}$
}

\begin{abstract}
RESUMO
A espirocercose, causada pelo parasito Spirocerca lupi, é considerada sub-clínica, sendo, na maioria das vezes, diagnosticada na necropsia. O exame endoscópio é o mais indicado no diagnóstico, mas a radiografia também pode auxiliar. O tratamento pode ser clínico ou cirúrgico. O presente trabalho tem o objetivo de descrever o tratamento cirúrgico de nódulos de Spirocerca lupi em um cão. Foi selecionada para fazer parte de uma pesquisa uma cadela sem raça definida, adulta. Foi realizado exame clínico e radiografia contrastada do esôfago com resultados normais. O animal foi submetido a esofagotomia torácica. Durante o trans-operatório foram constatados no esôfago grandes nódulos de Spirocerca lupi, os quais foram removidos. O esôfago foi suturado em pontos separados simples em plano único. A cavidade torácica foi fechada de forma rotineira. A dieta pós-operatória constou de líquidos por três dias, alimentos pastosos por mais três dias e a seguir ração comercial. A cadela foi acompanhada durante noventa dias e não apresentou alterações clínicas dignas de nota $\mathrm{O}$ animal se recuperou completamente. Concluiu-se que nódulos de Spirocerca lupi podem ser removidos cirurgicamente com êxito. O exame clínico e radiográfico não foram suficientes para diagnóstico da espirocercose no caso relatado.
\end{abstract}

Descritores: Spirorerca lupi, canino, esôfago, cirurgia, espirocercose.

\begin{abstract}
Spirocercosis, caused by Spirocerca lupi, is considered sub-clinic. In the most cases the diagnosis is made in necropsy. The endoscopic exam is more indicated in diagnosis but the radiography also can to be aid. The treatment can to be clinic or surgical. This paper describes a surgical treatment of the Spirocerca lupi nodules in a bitch. A mongrel adult bitch was chosen to announce in a research. Clinic exam and contrasted esophagic radiography were made with normal results. The animal was submitted a thoracic esophagotomy. During surgical procedure large nodules of Spirocerca lupi were observed, and removed. The esophagus was sutured in single layer with interrupted points. Thoracic wall was closed in usual manner. The postoperative diet was liquid for three days, soft food for three days, starting from commercial food. The bitch was accompanied during ninety days. During this time significant clinic alterations were not observed. Animal had complete recovery. In conclusion Spirocerca lupi nodules can to be removed surgically with success. Clinic exam and radiography was not sufficient to diagnose spirocercosis in this case.
\end{abstract}

Key words: Spirorerca lupi, canine, esophagus, surgery, spirocercosis. 


\section{INTRODUÇÃO}

A espirocercose é uma parasitose causada pelo nematódeo Spirocerca lupi [8] o qual produz granuloma esofágico [6]. É considerada subclínica e de difícil diagnóstico [12] por isso, na maioria das vezes, é diagnosticada na necropsia [11,12]. Para se detectar a enfermidade o exame mais eficaz é a endoscopia [9], mas os sinais radiográficos também podem ser indicativos $[4,5,9,10]$. O tratamento pode ser cirúrgico $[1,3]$ ou clínico [2,7]. O presente trabalho tem o objetivo de descrever a remoção cirúrgica bem sucedida de nódulos de Spirocerca lupi em um cão.

\section{RELATO DE CASO}

Foi selecionada para fazer parte de uma pesquisa uma cadela sem raça definida (S.R.D.), adulta, $18 \mathrm{~kg}$. A cadela foi submetida a exame clínico e foi realizada radiografia contrastada do esôfago. $\mathrm{O}$ animal foi anestesiado com halotano ${ }^{1}$. Foi feita toracotomia no sexto espaço intercostal. Foram constatados no esôfago três nódulos de Spirocerca lupi de aproximadamente $2 \mathrm{~cm}$ de diâmetro cada (Figura 1A). Os nódulos foram retirados (Figura 1B) e o esôfago foi suturado em pontos separados simples com fio de algodão (Figura1C). Após a cirurgia o animal recebeu dieta líquida durante três dias, dieta pastosa durante três dias e, a seguir, ração comercial. A cadela foi acompanhada clinicamente durante 90 dias.

\section{DISCUSSÃO}

Resolveu-se relatar este caso porque a literatura registra poucos casos de remoção cirúrgica de nódulos de Spirocerca lupi [1,3]. No exame clínico pré-operatório, não foram encontrados sinais clínicos da doença, reforçando o aspecto subclínico [11,12]. $\mathrm{Na}$ radiografia pré-operatória não foram encontradas alterações, embora seja considerada importante para diagnóstico da enfermidade [9,10]. $\mathrm{O}$ exame mais indicado seria a endoscopia [9], o qual não foi feito porque o equipamento não estava disponível. Pode ser indicado tratamento clínico [2,7], o qual não foi feito porque a espirocercose não foi detectada no pré-operatório. Os granulomas esofágicos, característicos da enfermidade [6] (Figura 1), só foram detectados quando o esôfago foi exposto, reforçando a afirmação de que muitas vezes a espirocercose só é diagnosticada em endoscopia [9] ou na necropsia [11,12]. Durante o pósoperatório o animal se recuperou bem. Os nódulos encontrados neste animal foram removidos cirurgica- mente com êxito. Os exames clínicos e radiográficos não foram suficientes para diagnóstico da enfermidade no caso relatado.

\section{NOTA INFORMATIVA}

${ }^{1}$ Halotano, Hoechst do Brasil, química e farmacêutica S.A., São Paulo, SP.
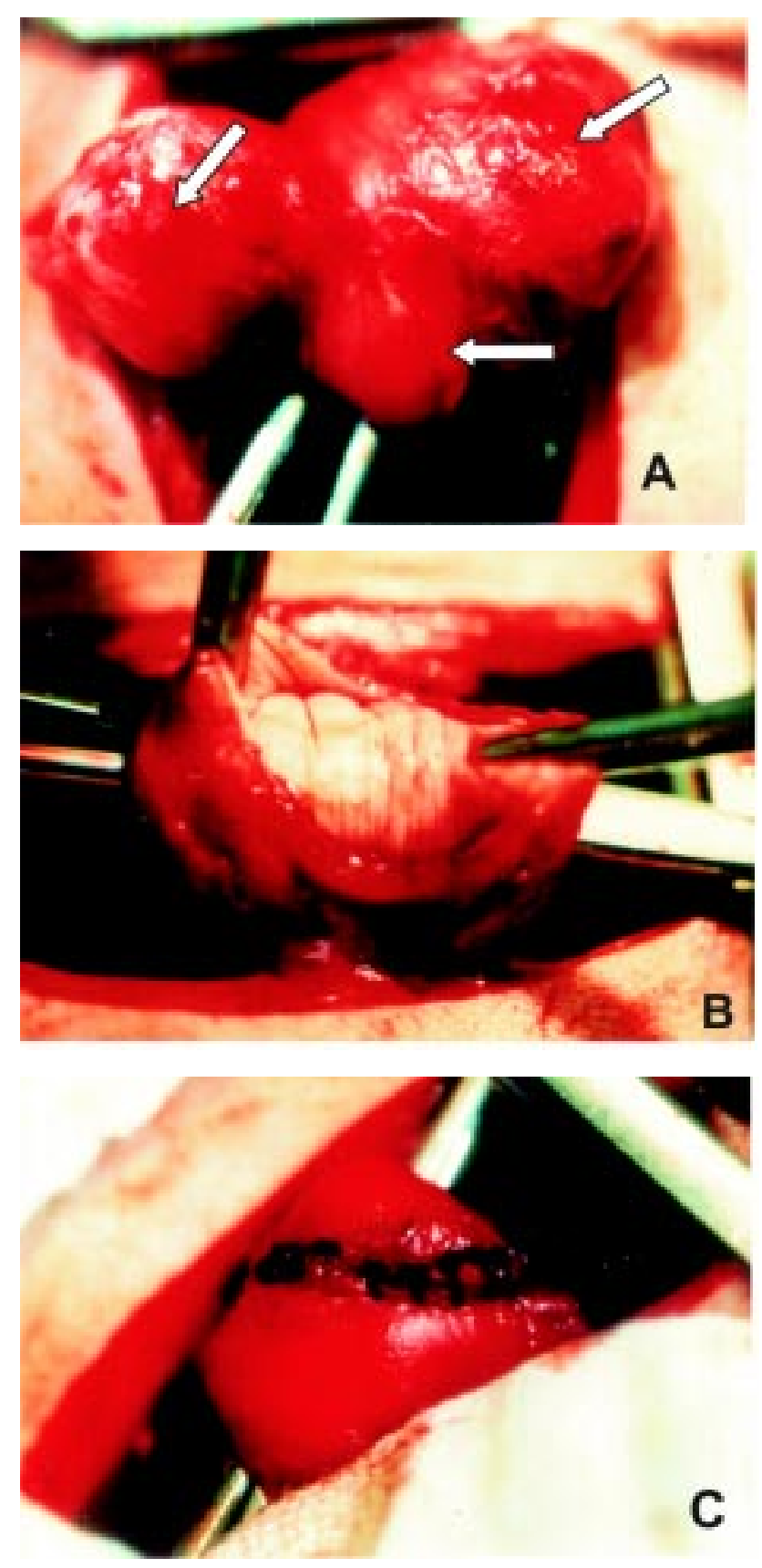

Figura 1.A: Cadela S.R.D. apresentando granuloma esofágico indicativo de espirocercose. Toracotomia no sexto espaço intercostal. Após toracotomia exposição do esôfago contendo os 3 nódulos de Spirocerca lupi de aproximadamente $2 \mathrm{~cm}$ de diâmetro cada (Setas). B: Abertura do esôfago para remoção dos nódulos. C: Esôfago suturado com pontos separados simples extra-mucosa com fio de algodão. 


\section{REFERÊNCIAS}

1 Alvarenga J., Iwasaki M., Stopiglia A.J. \& Barros P.S.M. 1984. Remoção cirúrgica de nódulos parasitários de Spirocerca lupi na porção torácica do esôfago de cães. Veterinária Brasileira. 2: 5-6.

2 Berry W.L. 2004. Spirocerca lupi Esophageal Granulomas in 7 dogs: resolution after treatment with Doramectin. Journal of Veterinary Internal Medicine. 14: 609-612.

3 Colgrove D.J. 1971. Transthoracic esophageal surgery for obstructive lesions caused by Spirocerca lupi in dogs. Journal of the American Veterinary Medical Association. 158: 2073-2076.

4 Dvir E., Kinberger R.M. \& Malleczek D. 2001. Radiographic and Computed thomographic changes and clinical presentation of spirocercosis in the dog. Veterinary Radiology \& Ultrasound. 42: 119-129.

5 Evans L.B. 1983. Clinical diagnosis of Spirocerca lupi infestation in dogs. Journal of South African Veterinary Association. 54: 189-191.

6 Kruinigen H.J.V. 1995. Gastrointestinal system. In: Carlton W.W. \& Mcgavin M.D. (Eds). Thomson's special veterinary pathology. 2nd. edn. St. Louis: Mosby, pp.1-80.

7 Lavy E., Harrus S., Mazaki-Tovi M., Bark H., Markovics A., Hagag A., Aizenberg I. \& Aroch I. 2003. Spirocerca lupi in dogs: prophylactic effect of doramectin. Veterinary Parasitology. 75: 217-222.

8 Mattos-Júnior D.G. 1999. Manual de helmintoses comuns em cães. Niterói: EdUFF,

9 Mazaki-Tovi M., Baneth G., Aroch I., Harrus S., Kass P.H., Ben-Ari T., Zur G., Aizenberg I., Bark H. \& Lavy E. 2002. Canine spirocercosis: clinical, diagnostic, pathologic, and epidemiologic characteristics. Veterinary Parasitology. 107: 235-250.

10 Meléndez R.D. \& Suárez-Pellín C. 2001. Spirocerca lupi and dogs: the role of nematodes in carcinogenesis. Trends in Parasitology. 17: 516-517.

11 O'Brien D.J. 1980. The esophagus. In: Anderson N.V. (Ed). Veterinary Gastroenterology. Philadelphia: Lea \& Febiger, pp.372-391.

12 Santos A. S. O., Silveira L.S., Lemos L.S., Moreira L., Silva A.C., Carvalho E.C.Q. \& Matos W.R. 2004. Aneurisma aórtico pela Espirocercose em Brasília. Revista Brasileira de Saúde e Produção Animal. 5: 25-30. 